\title{
A New Large Scale Instability in Rotating Stratified Fluids Driven by Small Scale Forces
}

\author{
Anatoly Tur ${ }^{1}$, Malik Chabane ${ }^{1}$, Vladimir Yanovsky ${ }^{2}$ \\ ${ }^{1}$ Université de Toulouse [UPS], Centre National de la Recherche Scientifique, \\ Institut de Recherche en Astrophysique et Planétologie, Toulouse, France \\ ${ }^{2}$ Institute for Single Crystals, National Academy of Science Ukraine, Kharkov, Ukraine \\ Email: anatoly.tour@irap.omp.eu
}

Received October 25, 2013; revised November 25, 2013; accepted December 3, 2013

Copyright (C) 2013 Anatoly Tur et al. This is an open access article distributed under the Creative Commons Attribution License, which permits unrestricted use, distribution, and reproduction in any medium, provided the original work is properly cited.

\begin{abstract}
In this paper, we find a new large scale instability displayed by a stratified rotating flow in forced turbulence. The turbulence is generated by a small scale external force at low Reynolds number. The theory is built on the rigorous asymptotic method of multi-scale development. There is no other special constraint concerning the force. In previous papers, the force was either helical or violating parity invariance. The nonlinear equations for the instability are obtained at the third order of the perturbation theory. In this article, we explain a detailed study of the linear stage of the instability.
\end{abstract}

Keywords: Large Scale Vortex Instability; Coriolis Forse; Buoyancy; Multi-Scale Development; Small Scale Turbulence

\section{Introduction}

Large scale instabilities are very important in fluid dynamics. They generate vortices which play a fundamental role in turbulence and in transport processes. The characteristic dimensions of the large scale structures are greater than the typical scale of the turbulence. The turbulence is often simulated using a small scale external force. In this case, the large scale vortices are much greater than the scale of the external force. Large scale vortices are well observed in planetary atmospheres [1, 2], in numerical simulations, and in laboratory experiments [3-10]. The generation process of large scale instabilities has been studied in several papers [11-19]. In these papers, the turbulence which generates these coherent large scale structures cannot be homogenous, isotropic, or mirror invariant. A series of papers have shown that the essential mechanism which leads to the generation of large scale vortices is the lack of reflection invariance. This mechanism was called the hydrodynamic $\alpha$-effect by analogy with the similar mechanism of generation of large scale magnetic fields.

Turbulence lacking reflection invariance is helical and a pseudo-scalar $\boldsymbol{v} \cdot \boldsymbol{r o t v} \neq 0$ appears. Nevertheless, the helicity of turbulence by itself can not generate large scale vortices. Other factors which lack reflection invariance are necessary, such as, for instance, compressibility $[16,19]$ or temperature gradients $[17,18]$. Large scale instability can also appear if the turbulence lacks parity invariance (AKA effect) [12]. The helicity of the turbulence can be defined in a phenomenological way, but helicity can also be generated by an internal mechanism like rotation or buoyancy $[13,15,20]$.

Large scale instabilities in a stratified rotating flow were studied in [21,22]. In [21], it was shown that a rotating incompressible flow with a constant temperature gradient can not display a large scale instability. In [22] the author presented large scale instabilities with a quadratic temperature gradient. In both papers, the authors used the functional averaging method. This method has some inconveniences. Especially it is impossible to make a strict hierarchy of orders as in perturbation theory. This means that it is impossible to identify the orders in which the instability appears and the ones in which it is absent. That is why the fact that the instability is absent when using the functional averaging method can not exclude its occurrence when using the rigorous asymptotic method of multi-scale development.

The occurrence of large scale instability in helical stratified turbulence was confirmed by the multi-scale de- 
velopment method in [23]. In that paper it was shown that the instability appears at the third order in the asymptotical development built on the small value of the Reynolds number. But in the first papers on this subject, using the functional averaging method, it was not clear in which order the instability would appear.

Direct numerical simulation of the Boussinesq Equation confirmed the existence of large scale vortex generation in stratified and rotating flows [24,25]. Sometimes the appearance of large scale vortex structures is accompanied by an inverse cascade of energy both in the threedimensional case (AKA-effect [26]), and in the quasi two-dimensional case $[4,7,9,10]$. One may say that the inverse cascade itself is also one of the mechanisms of the generation of large scale structures [5,27]. One of the important large scale instabilities in an incompressible fluid is the AKA effect (Anisotropic Kinetic Alpha effect) which was found in the work of Frisch, She and Sulem [11]. In this paper, the large scale instability appears under the impact of a small scale force in which parity is broken (with zero helicity). In a later paper [12], the inverse cascade of energy and the nonlinear mode of instability saturation were studied. Despite the fact that the broken parity is a more general notion than helicity, in fact, the helicity $\boldsymbol{v} \cdot \operatorname{rot} \boldsymbol{v} \neq 0$ is the widespread mechanism of symmetry breaking in hydrodynamical flows. The injection of a helical external force into a hydrodynamic system has been studied in several papers $[16,19]$. As a result, it was understood that a small scale turbulence that is able to generate large scale perturbations can not be simply homogeneous, isotropic, and helical [28], but must have additional special properties. In some cases, the existence of a large scale instability has been shown (a vortex dynamo or the hydrodynamic $\alpha$-effect). In the magneto hydrodynamics of a conductive fluid, the $\alpha$-effect is well known [29]. In particular, in [17] it was shown that a large scale instability exists in convective systems with small scale helical turbulence. These papers as well as the results of numerical modelling are described in detail in the review article [30], which is focused essentially on possible applications of these results to the issue of tropical cyclone origination. In this paper, we develop an analytical theory of the new large scale instability which generates large scale vortices in a stratified rotating flow with a constant temperature gradient under the action of a small scale external force which does not have any particular properties (especially it is nonhelical and it does not lack parity invariance). The force only maintains turbulent fluctuations. In other words, this force cannot display any instability. But the situation changes when both the Coriolis force and the buoyancy are added to this force. The joint action of these forces generates an internal helicity, which in turn generates an instability. The theory of this instability is developed rigourously using the method of asymptotic multi-scale development similar to what was done by Frisch, She and Sulem for the theory of the AKA effect [11]. This method allows finding the equations for large scale perturbations as the secular equations of perturbation theory, to calculate the Reynolds stress tensor and to find the instability. Our paper is organised as follows: In Section 2, we formulate the problem and the equations for the Coriolis force and the stratification in the Boussinesq approximation; In Section 3, we examine the principal scheme of the multi-scale development and we give the secular equations. In Section 4, we describe the calculations of the Reynolds stress. In Section 5, we discuss the instability and the conditions for its realization. The results obtained are discussed in the conclusions given in Section 6. The Reynolds stress and internal helicity are calculted in Appendices A and B, respectively.

\section{The Main Equations and Formulation of the Problem}

Let us consider the equations for the motion of an incompressible fluid with a constant temperature gradient in the Boussinesq approximation:

$$
\begin{aligned}
& \frac{\partial \boldsymbol{V}}{\partial t}+(\boldsymbol{V} \cdot \nabla) \boldsymbol{V}+2 \boldsymbol{\Omega} \times \boldsymbol{V} \\
& =-\frac{1}{\rho_{0}} \nabla P+v \Delta \boldsymbol{V}+g \beta T \boldsymbol{I}+\boldsymbol{F}_{0}, \\
& \frac{\partial T}{\partial t}+(\boldsymbol{V} \cdot \nabla) T=\chi \Delta T-V_{z} A, \\
& \nabla \cdot \boldsymbol{V}=0 .
\end{aligned}
$$

Here, $\boldsymbol{I}=(0,0,1), \quad \beta$ is the thermal expansion coefficient, $A=\frac{\mathrm{d} T_{0}}{\mathrm{~d} z}$ is the constant equilibrium gradient of the temperature, $\rho_{0}=$ Const., and $\nabla T_{0}=A \boldsymbol{I}$. The external force $\boldsymbol{F}_{0}$ has zero divergence. Let $\lambda_{0}, t_{0}, f_{0}, v_{0}$ be, respectively, the characteristic scale, time, amplitude of the external force, and velocity of our system. We choose the dimensionless variables

$$
\begin{aligned}
& (t, \boldsymbol{x}, \boldsymbol{V}): \\
& \boldsymbol{x} \rightarrow \frac{\boldsymbol{x}}{\lambda_{0}}, t \rightarrow \frac{t}{t_{0}}, \boldsymbol{V} \rightarrow \frac{\boldsymbol{V}}{v_{0}}, \\
& \boldsymbol{F}_{0} \rightarrow \frac{\boldsymbol{F}_{0}}{f_{0}}, P \rightarrow \frac{P}{\rho_{0} P_{0}}, \\
& t_{0}=\frac{\lambda_{0}^{2}}{v}, P_{0}=\frac{v_{0} v}{\lambda_{0}}, f_{0}=\frac{v_{0} v}{\lambda_{0}^{2}}, v_{0}=\frac{f_{0} \lambda_{0}^{2}}{v} .
\end{aligned}
$$

Then, 


$$
\begin{aligned}
& \frac{\partial \boldsymbol{V}}{\partial t}+R(\boldsymbol{V} \cdot \nabla) \boldsymbol{V}+D \boldsymbol{l} \times \boldsymbol{V} \\
& =-\nabla P+\Delta \boldsymbol{V}+\left(\frac{\lambda_{0}^{2}}{v_{0} v}\right) g \beta T \boldsymbol{l}+\boldsymbol{F}_{0}, \\
& \frac{\partial T}{\partial t}+R(\boldsymbol{V} \cdot \nabla) T=\frac{1}{\operatorname{Pr}} \Delta T-R V_{z}\left(A \lambda_{0}\right),
\end{aligned}
$$

where $R=\frac{\lambda_{0} v_{0}}{v}, D=\sqrt{T a}$ where $R$ and $T a=\frac{4 \Omega^{2} \lambda_{0}^{4}}{v^{2}}$ are respectively the Reynolds number and the Taylor number on scale $\lambda_{0} \cdot \operatorname{Pr}=\frac{v}{\chi}$ represents the Prandtl number. We introduce the dimensionless temperature $T \rightarrow \frac{T}{\lambda_{0} A}$, and obtain the system of equations

$$
\begin{aligned}
& \frac{\partial \boldsymbol{V}}{\partial t}+R(\boldsymbol{V} \cdot \nabla) \boldsymbol{V}-\Delta \boldsymbol{V}+D \boldsymbol{l} \times \boldsymbol{V} \\
& =-\nabla P+\frac{R a}{R \operatorname{Pr}} T \boldsymbol{l}+\boldsymbol{F}_{0} \\
& \frac{1}{R}\left(\frac{\partial T}{\partial t}-\frac{1}{\operatorname{Pr}} \Delta T\right)=-V_{z}-(\boldsymbol{V} \cdot \nabla) T .
\end{aligned}
$$

Here, $R a=\frac{\lambda_{0}^{4} A g \beta}{\chi v}$ is the Rayleigh number on the scale $\lambda_{0}$. Furthermore, for the purpose of simplification, we will consider the case $\operatorname{Pr}=1$. We pass to the new temperature $T \rightarrow \frac{T}{R}$, and obtain

$$
\begin{aligned}
& \frac{\partial \boldsymbol{V}}{\partial t}+R(\boldsymbol{V} \cdot \nabla) \boldsymbol{V}-\Delta \boldsymbol{V}+D \boldsymbol{l} \times \boldsymbol{V} \\
& =-\nabla P+R a T \boldsymbol{l}+\boldsymbol{F}_{0}, \\
& \left(\frac{\partial T}{\partial t}-\Delta T\right)=-V_{z}-R(\boldsymbol{V} \cdot \nabla) T, \\
& \nabla \cdot \boldsymbol{V}=0 .
\end{aligned}
$$

We will consider as a small parameter of an asymptotic development the Reynolds number $R=\frac{\lambda_{0} v_{0}}{v} \ll 1$ on the scale $\lambda_{0}$. Concerning the parameters $R a$ and $D$, we do not choose any range of values for the moment. Let us examine the following formulation of the problem. We consider the external force as being small and of high frequency. This force leads to small scale fluctuations in velocity and temperature against a background of equilibrium. After averaging, these quickly oscillating fluctuations vanish. Nevertheless, due to small nonlinear interactions in some orders of perturbation theory, nonzero terms can occur after averaging. This means that they are not oscillatory, that is to say, they are large scale. From a formal point of view, these terms are secular, i.e., they create the conditions for the solvability of a large scale asymptotic development. So the purpose of this paper is to find and study the solvability equations, i.e., the equations for large scale perturbations. Let us denote the small scale variables by $x_{0}=\left(x_{0}, t_{0}\right)$, and the large scale ones by $X=(\boldsymbol{X}, T)$. The small scale partial derivative operation $\frac{\partial}{\partial x_{0}^{i}}, \frac{\partial}{\partial t_{0}}$, and the large scale ones $\frac{\partial}{\partial \boldsymbol{X}}, \frac{\partial}{\partial T}$ are written, respectively, as $\partial_{i}, \partial_{t}, \nabla_{i}$ and $\partial_{T}$. To construct a multi-scale asymptotic development we follow the method which is proposed in [11].

\section{The Multi-Scale Asymptotic Development}

Let us search for the solution to Equations (4) and (5) in the following form:

$$
\begin{aligned}
\boldsymbol{V}(\boldsymbol{x}, t) & =\frac{1}{R} \boldsymbol{W}_{-1}(X)+\boldsymbol{v}_{0}\left(x_{0}\right)+R \boldsymbol{v}_{1}+R^{2} \boldsymbol{v}_{2} \\
& +R^{3} \boldsymbol{v}_{3}+\cdots, \\
T(\boldsymbol{x}, t) & =\frac{1}{R} T_{-1}(X)+T_{0}\left(x_{0}\right) \\
& +R T_{1}+R^{2} T_{2}+R^{3} T_{3}+\cdots, \\
P(\boldsymbol{x}, t)= & \frac{1}{R^{3}} P_{-3}(X)+\frac{1}{R^{2}} P_{-2}(X)+\frac{1}{R} P_{-1}(X) \\
& +P_{0}\left(x_{0}\right)+R\left(P_{1}+\bar{P}_{1}(X)\right) \\
& +R^{2} P_{2}+R^{3} P_{3}+\cdots,
\end{aligned}
$$

Let us introduce the following equalities: $\boldsymbol{X}=R^{2} \boldsymbol{x}_{0}$ and $T=R^{4} t_{0}$ which lead to the expression for the space and time derivatives:

$$
\begin{aligned}
& \frac{\partial}{\partial x^{i}}=\partial_{i}+R^{2} \nabla_{i}, \\
& \frac{\partial}{\partial t}=\partial_{t}+R^{4} \partial_{T}, \\
& \frac{\partial^{2}}{\partial x^{j} \partial x^{j}}=\partial_{j j}+2 R^{2} \partial_{j} \nabla_{j}+R^{4} \partial_{j j} .
\end{aligned}
$$

Using indicial notation, the system of the equation can be written as

$$
\begin{aligned}
& \left(\partial_{t}+R^{4} \partial_{T}\right) V^{i}+R\left(\partial_{j}+R^{2} \nabla_{j}\right)\left(V^{i} V^{j}\right) \\
& +D \varepsilon_{i j k} l^{j} V^{k}=\left(\partial_{j}+R^{2} \nabla_{j}\right) P+ \\
& +\left(\partial_{j j}+2 R^{2} \partial_{j} \nabla_{j}+R^{4} \nabla_{j j}\right) V^{i}+R a T l^{i}+F_{0}^{i}, \\
& \partial_{t} T-\partial_{j j} T=-V^{z}-R \partial_{j}\left(V^{i} T\right), \\
& \left(\partial_{i}+R^{2} \nabla_{i}\right) V^{i}=0 .
\end{aligned}
$$

Substituting these expressions into the initial equations 
(4) and (5) and then gathering together the terms of the same order, we obtain the equations of the multi- scale asymptotic development and write down the obtained equations up to order $R^{3}$ inclusive. In the order $R^{-3}$ there is only the Equation

$$
\partial_{i} P_{-3}=0, \Rightarrow P_{-3}=P_{-3}(X) .
$$

In order $R^{-2}$ we have the equation

$$
\partial_{i} P_{-2}=0, \Rightarrow P_{-2}=P_{-2}(X) \text {. }
$$

In order $R^{-1}$ we get a system of equations:

$$
\begin{aligned}
& \partial_{t} W_{-1}^{i}-\partial_{j j} W_{-1}^{i}+D \varepsilon_{i j k} l^{j} W_{-1}^{k} \\
& =-\left(\partial_{i} P_{-1}+\nabla_{i} P_{-3}\right)+R a T_{-1} l^{i}-\partial_{j} W_{-1}^{i} W_{-1}^{j}, \\
& =-\left(\partial_{i} P_{-1}+\nabla_{i} P_{-3}\right)+R a T_{-1} l^{i}-\partial_{j} W_{-1}^{i} W_{-1}^{j}, \\
& \partial_{t} T_{-1}-\partial_{j j} T_{-1}=-\partial_{j} W_{-1}^{j} T_{-1}-W_{-1}^{z}, \\
& \partial_{i} W_{-1}^{i}=0 .
\end{aligned}
$$

The system of Equations (17) and (18) gives the secular terms

$$
-\nabla_{i} P_{-3}+R a T_{-1} l^{i}=D \varepsilon_{i j k} l^{j} W_{-1}^{k},
$$

which corresponds to a geostrophic equilibrum Equation, and

$$
W_{-1}^{z}=0 \text {. }
$$

In zero order $R^{0}$, we have the following system of equations:

$$
\begin{aligned}
& \partial_{t} v_{0}^{i}-\partial_{j j} v_{0}^{i}+\partial_{j}\left(W_{-1}^{i} v_{0}^{j}+v_{0}^{i} W_{-1}^{j}\right)+D \varepsilon_{i j k} l^{j} v_{0}^{k} \\
& =-\left(\partial_{i} P_{0}+\nabla_{i} P_{-2}\right)+R a T_{0} l^{i}+F_{0}^{i}, \\
& \partial_{t} T_{0}-\partial_{j j} T_{0}+\partial_{j}\left(W_{-1}^{j} T_{0}+v_{0}^{j} T_{-1}\right)=-v_{0}^{z}, \\
& \partial_{i} v_{0}^{i}=0 .
\end{aligned}
$$

These equations give one secular Equation:

$$
\nabla P_{-2}=0, \Rightarrow P_{-2}=\text { Const. }
$$

Let us consider the equations of the first approximation $R$ :

$$
\begin{aligned}
& \partial_{t} v_{1}^{i}-\partial_{j j} v_{1}^{i}+D \varepsilon_{i j k} l^{j} v_{1}^{k}+\partial_{j}\left(W_{-1}^{i} v_{1}^{j}+v_{1}^{i} W_{-1}^{j}+v_{0}^{i} v_{0}^{j}\right) \\
& =-\nabla_{j}\left(W_{-1}^{i} W_{-1}^{j}\right)-\left(\partial_{i} P_{1}+\nabla_{i} P_{-1}\right)+R a T_{1} l^{i}, \\
& \partial_{t} T_{1}-\partial_{j j} T_{1}+\partial_{j}\left(W_{-1}^{j} T_{1}+v_{1}^{j} T_{-1}+v_{0}^{j} T_{0}\right) \\
& +\nabla_{j}\left(W_{-1}^{j} T_{-1}\right)=-v_{1}^{z}, \\
& \partial_{i} V_{1}^{i}+\nabla_{i} W_{-1}^{i}=0 .
\end{aligned}
$$

From this system of equations there follows the secular equations:

$$
\begin{aligned}
& \nabla_{i} W_{-1}^{i}=0, \\
& \nabla_{j}\left(W_{-1}^{i} W_{-1}^{j}\right)=-\nabla_{i} P_{-1}, \\
& \nabla_{j}\left(W_{-1}^{j} T_{-1}\right)=0 .
\end{aligned}
$$

The secular equations (27) and (29) are satisfied by choosing the following geometry for the velocity field:

$$
\begin{aligned}
& \boldsymbol{W}=\left(W_{-1}^{x}(Z), W_{-1}^{y}(Z), 0\right) ; T_{-1}=T_{-1}(Z) ; \\
& \nabla P_{-1}=0, \Rightarrow P_{-1}=\text { Const. }
\end{aligned}
$$

In the second order $R^{2}$, we obtain the equations

$$
\begin{aligned}
& \partial_{t} v_{2}^{i}-\partial_{j j} v_{2}^{i}-2 \partial_{j} \nabla_{j} v_{0}^{i} \\
& +\partial_{j}\left(W_{-1}^{i} v_{2}^{j}++v_{2}^{i} W_{-1}^{j}+v_{0}^{i} v_{1}^{j}+v_{1}^{i} v_{0}^{j}\right)+D \varepsilon_{i j k} l^{j} v_{2}^{k} \\
& =-\nabla_{j}\left(W_{-1}^{i} v_{0}^{j}+v_{0}^{i} W_{-1}^{j}\right)-\left(\partial_{i} P_{2}+\nabla_{i} P_{0}\right)+R a T_{2} l^{i}, \\
& \partial_{t} T_{2}-\partial_{j j} T_{2}-2 \partial_{j} \nabla_{j} T_{0} \\
& +\partial_{j}\left(W_{-1}^{j} T_{2}+v_{2}^{j} T_{-1}+v_{0}^{j} T_{1}+v_{1}^{j} T_{0}\right) \\
& =-\nabla_{j}\left(W_{-1}^{j} T_{0}+v_{0}^{j} T_{-1}\right)-v_{2}^{z}, \\
& \partial_{i} v_{2}+\nabla_{i} v_{0}=0 .
\end{aligned}
$$

It is easy to see that there are no secular terms in this order..

Let us come now to the most important order $R^{3}$. In this order we obtain the equations

$$
\begin{aligned}
& \partial_{t} v_{3}^{i}+\partial_{T} W_{-1}^{i}-\left(\partial_{j j} v_{3}^{i}+2 \partial_{j} \nabla_{j} v_{1}^{i}+\nabla_{j j} W_{-1}^{i}\right) \\
& +\nabla_{j}\left(W_{-1}^{i} v_{1}^{j}+v_{1}^{i} W_{-1}^{j}+v_{0}^{i} v_{0}^{j}\right) \\
& +\partial_{j}\left(W_{-1}^{i} v_{3}^{j}+v_{3}^{i} W_{-1}^{j}+v_{0}^{i} v_{2}^{j}+v_{2}^{i} v_{0}^{j}+v_{1}^{i} v_{1}^{j}\right)+D \varepsilon_{i j k} l^{j} v_{3}^{k} \\
& =-\left(\partial_{i} P_{3}+\nabla_{i} \bar{P}_{1}\right)+R a T_{3} l^{i}, \\
& \partial_{t} T_{3}+\partial_{T} T_{-1}-\left(\partial_{j j} T_{3}+2 \partial_{j} \nabla_{j} T_{1}+\nabla_{j j} T_{-1}\right) \\
& +\nabla_{j}\left(W_{-1}^{j} T_{1}+v_{1}^{j} T_{-1}+v_{0}^{j} T_{0}\right)+ \\
& +\partial_{j}\left(W_{-1}^{j} T_{3}+v_{3}^{j} T_{-1}+v_{0}^{j} T_{2}+v_{2}^{j} T_{0}+v_{1}^{j} T_{1}\right)=-v_{3}^{z}, \\
& \partial_{i} v_{3}+\nabla_{i} v_{1}=0 .
\end{aligned}
$$

From this we get the main secular Equation:

$$
\begin{aligned}
& \partial_{T} W_{-1}^{i}-\Delta W_{-1}^{i}+\nabla_{k}\left(\overline{v_{0}^{k} v_{0}^{i}}\right)=-\nabla_{i} \bar{P}_{1}, \\
& \partial_{T} T_{-1}-\Delta T_{-1}+\nabla_{k}\left(\overline{v_{0}^{k} T_{0}}\right)=0 .
\end{aligned}
$$

There is also an Equation to find the pressure $P_{-3}$ :

$$
-\nabla_{i} P_{-3}+R a T_{-1} l^{i}=D \varepsilon_{i j k} l^{j} W_{-1}^{k} .
$$

\section{Calculations of the Reynolds Stresses}

It is clear that the essential Equation for finding the non- 
linear alpha-effect is Equation (36). In order to obtain these equations in closed form, we need to calculate the Reynolds stresses $\nabla_{k}\left(\overline{v_{0}^{k} v_{0}^{i}}\right)$. First of all we have to calculate the fields of zero approximation $v_{0}^{k}$. From the asymptotic development in zero order we have

$$
\begin{aligned}
& \partial_{t} v_{0}^{i}-\partial_{j j} v_{0}^{i}+W_{-1}^{k} \partial_{k} v_{0}^{i}+D \varepsilon_{i j k} l^{j} v_{0}^{k} \\
& =-\partial_{i} P_{0}+R a T_{0} l^{i}+F_{0}^{i}, \\
& \partial_{t} T_{0}-\partial_{j j} T_{0}+W_{-1}^{k} \partial_{k} T_{0}=-v_{0}^{k} l^{k} .
\end{aligned}
$$

Let us introduce the operator $\hat{D}_{0}$ :

$$
\hat{D}_{0} \equiv \partial_{t}-\partial_{j j}+W^{k} \partial_{k} \text {. }
$$

Using $\hat{D}_{0}$, we rewrite Equations (39) and (40):

$$
\begin{aligned}
& \hat{D}_{0} v_{0}^{i}+D \varepsilon_{i j k} l^{j} v_{0}^{k}=-\partial_{i} P_{0}+R a T_{0} l^{i}+F_{0}^{i}, \\
& \hat{D}_{0} T_{0}=-v_{0}^{k} l^{k} .
\end{aligned}
$$

Eliminating the temperature and pressure from Equation (42), we obtain

$$
\left(\hat{D}_{0}^{2} \delta_{i k}+\hat{P}_{i p} R a l^{p} l^{k}+D \hat{D}_{0} \hat{P}_{i p} \varepsilon_{p j k} l^{j}\right) v_{0}^{k}=\hat{D}_{0} F_{0}^{i} .
$$

Here, $\hat{P}_{i p}$ is the projection operator

$$
\hat{P}_{i p} \equiv \delta_{i p}-\frac{\partial_{i} \partial_{p}}{\partial_{j j}} .
$$

Dividing this equation by $\hat{D}_{0}^{2}$, we can write it in the form

$$
M_{i k} v_{0}^{k}=\frac{F_{0}^{i}}{\hat{D}_{0}},
$$

where $M_{i k}$ is the operator given by

$$
M_{i k} \equiv \delta_{i k}+R a \frac{\hat{P}_{i p}}{\hat{D}_{0}^{2}} l^{p} l^{k}+D \frac{\hat{P}_{i p}}{\hat{D}_{0}} \varepsilon_{p j k} l^{j} .
$$

We must now determine the inverse operator $M_{k j}^{-1}$, i.e., : $M_{i k} M_{k j}^{-1}=\delta_{i j}$.

After some calculation, we find

$$
\begin{aligned}
M_{k j}^{-1} & =\frac{1}{\Psi}\left[\left(1+\frac{R a}{\hat{D}_{0}^{2}} \hat{P}_{33}\right) \delta_{k j}+D \frac{\hat{P}_{k p}}{\hat{D}_{0}} \varepsilon_{p j n} l^{n}\right. \\
& \left.-R a \frac{\hat{P}_{k p}}{\hat{D}_{0}^{2}} l^{p} l^{j}+\xi_{k j}\right] .
\end{aligned}
$$

Here,

$$
\begin{aligned}
\Psi & =1+\frac{D^{2}}{\hat{D}_{0}^{2}} \hat{P}_{11}+\frac{R a}{\widehat{D}_{0}^{2}} \hat{P}_{33}+\frac{D^{2} R a}{\hat{D}_{0}^{4}}\left(\hat{P}_{11} \hat{P}_{33}-\hat{P}_{13}^{2}\right) \\
& =\operatorname{det}(M)
\end{aligned}
$$

and

$$
\xi=\left(\begin{array}{ccc}
0 & \frac{D R a}{\hat{D}_{0}^{3}}\left(\hat{P}_{11} \hat{P}_{33}-\hat{P}_{13}^{2}\right) & 0 \\
-\frac{D R a}{\hat{D}_{0}^{3}} \hat{P}_{33} & 0 & \frac{D R a}{\hat{D}_{0}^{3}} \hat{P}_{13} \\
-\frac{D^{2}}{\hat{D}_{0}^{2}} \hat{P}_{13} & 0 & \frac{D^{2}}{\hat{D}_{0}^{2}} \hat{P}_{11}
\end{array}\right)
$$

Consequently, the expression for the velocity $v_{0}^{k}$ takes the form

$$
\begin{aligned}
v_{0}^{k} & =\frac{1}{\Psi}\left[\left(1+\frac{R a \hat{P}_{33}}{\hat{D}_{0}^{2}}\right) \delta_{k j}+D \frac{\hat{P}_{k p}}{\hat{D}_{0}} \varepsilon_{p j n} l^{n}\right. \\
& \left.-R a \frac{\hat{P}_{k p}}{\hat{D}_{0}^{2}} l^{p} l^{j}+\xi_{k j}\right] \frac{F_{0}^{j}}{\hat{D}_{0}} .
\end{aligned}
$$

In order to use these formulas, we have to specify in explicit form the external force $F_{0}^{j}$. Let us specify it by

$$
\boldsymbol{F}_{0}=f_{0}\left(\boldsymbol{i} \cos \varphi_{1}+\boldsymbol{k} \cos \varphi_{2}\right),
$$

where

$$
\varphi_{1}=k_{0} z-\omega_{0} t, \varphi_{2}=k_{0} x-\omega_{0} t,
$$

or

$$
\begin{aligned}
& \varphi_{1}=\boldsymbol{k}_{1} \cdot \boldsymbol{x}-\omega_{0} t, \varphi_{2}=\boldsymbol{k}_{2} \cdot \boldsymbol{x}-\omega_{0} t, \\
& \boldsymbol{k}_{1}=k_{0}(0,0,1) ; \boldsymbol{k}_{2}=k_{0}(1,0,0) .
\end{aligned}
$$

One can check that $\nabla \cdot \boldsymbol{F}_{0}=0$ and $\boldsymbol{F}_{0} \cdot \nabla \times \boldsymbol{F}_{0}=0$

Formulas (50) and (52) allow us to easily make intermediate calculations, but in the final formulas we obviously shall take $f_{0}, k_{0}$ and $\omega_{0}$ as equal to unity, since the external force is dimensionless and depends only on the dimensionless arguments of space and time. The force (50) is physically simple and can be realized in laboratory experiments and in numerical simulations.

The force (50) can be written in complex form:

$$
\begin{aligned}
\boldsymbol{F}_{0}= & \boldsymbol{A} \exp \left(i \varphi_{1}\right)+\boldsymbol{A}^{*} \exp \left(-i \varphi_{1}\right) \\
& +\boldsymbol{B} \exp \left(i \varphi_{2}\right)+\boldsymbol{B}^{*} \exp \left(-i \varphi_{2}\right),
\end{aligned}
$$

where $\boldsymbol{A}$ and $\boldsymbol{B}$ have the forms

$$
\boldsymbol{A}=\frac{f_{0}}{2} \boldsymbol{i}, \boldsymbol{B}=\frac{f_{0}}{2} \boldsymbol{k} .
$$

The effect of the operator $\hat{D}_{0}$ on the proper function $\exp [i(\boldsymbol{k} \cdot \boldsymbol{x}-\omega t)]$ has obviously the form

$$
\hat{D}_{0} \exp [i(\boldsymbol{k} \cdot \boldsymbol{x}-\omega t)]=\hat{D}_{0}(\omega, \boldsymbol{k}) \exp [i(\boldsymbol{k} \cdot \boldsymbol{x}-\omega t)],
$$

where $\hat{D}_{0}(\omega, \boldsymbol{k})$ is

$$
\hat{D}_{0}(\omega, \boldsymbol{k})=i(\omega+\boldsymbol{k} \cdot \boldsymbol{W})+k^{2} .
$$

From this it follows that

$$
\hat{D}_{0}\left(\omega,-\boldsymbol{k}_{1}\right)=i\left(\omega-\boldsymbol{k}_{1} \cdot \boldsymbol{W}\right)+k_{1}^{2},
$$




$$
\begin{aligned}
& \hat{D}_{0}^{*}\left(\omega,-\boldsymbol{k}_{1}\right)=\hat{D}_{0}\left(-\omega, \boldsymbol{k}_{1}\right), \\
& \hat{D}_{0}\left(\omega,-\boldsymbol{k}_{2}\right)=i\left(\omega-\boldsymbol{k}_{2} \cdot \boldsymbol{W}\right)+k_{2}^{2}, \\
& \hat{D}_{0}^{*}\left(\omega,-\boldsymbol{k}_{2}\right)=\hat{D}_{0}\left(-\omega, \boldsymbol{k}_{2}\right) .
\end{aligned}
$$

From Formulas (49) and (53), it follows that the field $v_{0}^{k}$ is composed of four terms:

$$
v_{0}^{k}=v_{01}^{k}+v_{02}^{k}+v_{03}^{k}+v_{04}^{k},
$$

where

$$
v_{02}^{k}=\left(v_{01}^{k}\right)^{*}, v_{04}^{k}=\left(v_{03}^{k}\right)^{*} .
$$

Finally, we introduce the notation

$$
\begin{aligned}
& \hat{D}_{0}\left(\omega_{0},-\boldsymbol{k}_{1}\right)=1+i\left(1-W_{1}\right) \equiv D_{1}, \\
& \hat{D}_{0}\left(-\omega_{0}, \boldsymbol{k}_{1}\right)=D_{1}^{*}, \\
& \hat{D}_{0}\left(\omega_{0},-\boldsymbol{k}_{2}\right)=1+i\left(1-W_{2}\right) \equiv D_{2}, \\
& \hat{D}_{0}\left(-\omega_{0}, \boldsymbol{k}_{2}\right)=D_{2}^{*},
\end{aligned}
$$

where $\left(W_{1}, W_{2}\right) \equiv\left(W^{x}, W^{y}\right)$. Taking into account these formulas, we can write down the velocities $v_{0}^{k}$ in the form

$$
\begin{aligned}
& v_{01}^{k}=M_{k j(1)}^{-1} \frac{A^{j}}{D_{1}^{*}} \mathrm{e}^{i \varphi_{1}}, \\
& v_{03}^{k}=M_{k j(2)}^{-1} \frac{B^{j}}{D_{2}^{*}} \mathrm{e}^{i \varphi_{2}},
\end{aligned}
$$

where

$$
\begin{aligned}
& M_{k j(1)}^{-1} \\
& =\frac{1}{\Psi_{(1)}}\left[\left(1+\frac{R a \hat{P}_{33}}{D_{1}^{* 2}}\right) \delta_{k j}+D \frac{\hat{P}_{k p}}{D_{1}^{*}} \varepsilon_{p j n} l^{n}-R a \frac{\hat{P}_{k p}}{D_{1}^{* 2}} l^{p} l^{j}+\xi_{k j(1)}\right]
\end{aligned}
$$

and

$$
\begin{aligned}
& M_{k j(2)}^{-1} \\
& =\frac{1}{\Psi_{(2)}}\left[\left(1+\frac{R a \hat{P}_{33}}{D_{2}^{* 2}}\right) \delta_{k j}+D \frac{\hat{P}_{k p}}{D_{2}^{*}} \varepsilon_{p j n} l^{n}-R a \frac{\hat{P}_{k p}}{D_{2}^{* 2}} l^{p} l^{j}+\xi_{k j(2)}\right]
\end{aligned}
$$

We can now calculate the Reynolds stresses:

$$
\overline{v_{0}^{k} v_{0}^{i}}=2 \operatorname{Re}\left(\overline{v_{01}^{k} v_{01}^{i *}}+\overline{v_{03}^{k^{*}} v_{03}}\right),
$$

which can be decomposed into two components:

$$
\overline{v_{0}^{k} v_{0}^{i}}=T_{(1)}^{k i}+T_{(2)}^{k i},
$$

where $T_{(1)}^{k i}$ and $T_{(2)}^{k i}$ can be expressed as follows:

$$
\begin{aligned}
T_{(1)}^{k i} & =\overline{v_{01}^{k} v_{01}^{i *}}+\overline{v_{01}^{k *} v_{01}^{i}}, \\
T_{(2)}^{k i} & =\overline{v_{03}^{k} v_{03}^{i *}}+\overline{v_{03}^{k *} v_{03}^{i}} .
\end{aligned}
$$

Taking into account Formulas (64) and (65), we obtain

$$
\hat{P}_{11}=\frac{1}{2}, \hat{P}_{33}=\frac{1}{2}, \hat{P}_{13}=-\frac{1}{2}, \hat{P}_{i 2}=\delta_{i 2} .
$$

We can write down the components $T_{(1)}^{3 i}$ and $T_{(2)}^{3 i}$, which are the ones of interest:

$$
\begin{aligned}
& T_{(1)}^{31}=\frac{D^{2}\left(D_{1}^{2}+D_{1}^{* 2}+R a\right)}{8|\Psi|^{2}\left|D_{1}\right|^{6}}, \\
& T_{(2)}^{31}=\frac{R a\left(D_{2}^{2}+D_{2}^{* 2}+D^{2}\right)}{8|\Psi|^{2}\left|D_{2}\right|^{6}}, \\
& T_{(1)}^{32}=-\frac{D^{3}\left(R a+2\left|D_{1}\right|^{2}\right)}{8|\Psi|^{2}\left|D_{1}\right|^{8}}, \\
& T_{(2)}^{32}=-\frac{D R a\left(D_{2}^{3}+D_{2}^{* 3}+D^{2}\right)}{8|\Psi|^{2}\left|D_{2}\right|^{8}} .
\end{aligned}
$$

Finally, using the following relations (we have similar formulas for $D_{2}$ after replacing $W_{1}$ with $W_{2}$ ):

$$
\begin{aligned}
& D_{1}=1+i\left(1-W_{1}\right), \\
& \left|D_{1}\right|^{2}=1+\left(1-W_{1}\right)^{2}, \\
& D_{1}^{2}=1-\left(1-W_{1}\right)^{2}+2 i\left(1-W_{1}\right), \\
& \left|D_{1}\right|^{4}=\left[1+\left(1-W_{1}\right)^{2}\right]^{2}, \\
& D_{1}^{3}=1-3\left(1-W_{1}\right)^{2}+i\left[1-W_{1}-3\left(1-W_{1}\right)^{3}\right] \\
& \left|D_{1}\right|^{6}=\left[1+\left(1-W_{1}\right)^{2}\right]^{3}, \\
& \left|D_{1}\right|^{8}=\left[1+\left(1-W_{1}\right)^{2}\right]^{4}, \\
& \Psi_{(1)}=1+\frac{D^{2}}{2 D_{1}^{* 2}+\frac{R a}{2 D_{1}^{* 2}},} \\
& \left|\Psi_{(1)}\right|^{2}= \\
& \frac{4\left|D_{1}\right|^{4}+\left(2 D^{2}+2 R a\right)\left(D_{1}^{2}+D_{1}^{* 2}\right)+2 R a D^{2}+R a^{2}+D^{4}}{4\left|D_{1}\right|^{4}} .
\end{aligned}
$$

We can then express $T_{(1)}^{31}, T_{(2)}^{31}, T_{(1)}^{32}$ and $T_{(2)}^{32}$ :

$$
\begin{aligned}
& T_{(1)}^{31}=\frac{D^{2}\left[2+R a-2\left(1-W_{1}\right)^{2}\right]}{\Xi_{(1)}}, \\
& T_{(2)}^{31}=\frac{R a\left[2+D^{2}-2\left(1-W_{2}\right)^{2}\right]}{\Xi_{(2)}},
\end{aligned}
$$




$$
\begin{aligned}
T_{(1)}^{32} & =\frac{-D^{3}\left[R a+2\left[1-\left(1-W_{1}\right)^{2}\right]\right]}{\Xi_{(1)}}, \\
T_{(2)}^{32} & =\frac{-D R a\left[2+D^{2}-6\left(1-W_{2}\right)^{2}\right]}{\Xi_{(2)}},
\end{aligned}
$$

where

$$
\begin{aligned}
& \Xi_{(1),(2)}=2\left[D^{4}+R a^{2}+2 D^{2} R a+4\left[1+\left(1-W_{1,2}\right)^{2}\right]^{2}\right. \\
& \left(2 D^{2}+2 R a\right)\left[2-2\left(1-W_{1,2}\right)^{2}\right]\left[1+\left(1-W_{1,2}\right)^{2}\right] .
\end{aligned}
$$

\section{Large Scale Instability}

Let us write down in the explicit form the equations for nonlinear instability:

$$
\begin{aligned}
& \partial_{T} W_{1}+\nabla_{Z} T_{(1)}^{31}+\nabla_{Z} T_{(2)}^{31}=\Delta W_{1}, \\
& \partial_{T} W_{2}+\nabla_{Z} T_{(1)}^{32}+\nabla_{Z} T_{(2)}^{32}=\Delta W_{2},
\end{aligned}
$$

where the components $T_{(1)}^{31}, T_{(2)}^{31}, T_{(1)}^{32}$ and $T_{(2)}^{32}$ of the Reynolds stress tensor are as defined in the previous section.

One can see that for small values of the variables $W_{1}$ and $W_{2}$, Equations (83) and (84) are reduced to linear equations and describe the linear stage of instability:

$$
\begin{aligned}
& \partial_{T} W_{1}+a \nabla_{Z} W_{1}+b \nabla_{Z} W_{2}=\Delta W_{1}, \\
& \partial_{T} W_{2}+c \nabla_{Z} W_{1}+d \nabla_{Z} W_{2}=\Delta W_{2},
\end{aligned}
$$

where the coeficients $a, b, c$ and $d$ can be written as

$$
a=\frac{a^{\prime}}{\Lambda} b=\frac{b^{\prime}}{\Lambda} c=\frac{c^{\prime}}{\Lambda} d=\frac{d^{\prime}}{\Lambda}
$$

with

$$
\begin{aligned}
a^{\prime} & =4 D^{6}+64 D^{2}+D^{2} R a^{3}+\left(2 D^{4}-4 D^{2}\right) R a^{2} \\
& +\left(D^{6}+48 D^{2}\right) R a, \\
b^{\prime} & =\left(D^{2}+4\right) R a^{3}+2 D^{4} R a^{2} \\
& +\left(D^{6}-4 D^{4}+48 D^{2}+64\right) R a, \\
c^{\prime} & =-2 D^{7}+16 D^{5}-96 D^{3}-D^{3} R a^{3}+ \\
& +\left(2 D^{3}-2 D^{5}\right) R a^{2}-\left(D^{7}+16 D^{3}\right) R a, \\
d^{\prime} & =-\left(D^{3}+2 D\right) R a^{3}-\left(2 D^{5}+16 D\right) R a^{2} \\
& +\left(-D^{7}+2 D^{5}-48 D^{3}+32 D\right) R a,
\end{aligned}
$$

and

$$
\Lambda=4\left(D^{4}+R a^{2}+2 R a D^{2}+16\right)^{2},
$$

which are the explicit forms of the quite bulky coeffi- cients. However, these coefficients can be expressed using the internal helicity $H_{0}$ of the velocity field $v_{0}$, calculated in Appendix B.

$$
H_{0}^{2}=\frac{\left[-16 D-D R a^{2}+\left(D^{3}+4 D\right) R a\right]^{2}}{4 \Lambda} .
$$

Therefore, we can write the constant coefficients $a, b, c$ and $d$ with respect to $H_{0}$ :

$$
a=H_{0}^{2} \frac{4 a^{\prime}}{\Pi}, b=H_{0}^{2} \frac{4 b^{\prime}}{\Pi}, c=H_{0}^{2} \frac{4 c^{\prime}}{\Pi}, d=H_{0}^{2} \frac{4 d^{\prime}}{\Pi},
$$

where $\Pi=\left[-16 D-D R a^{2}+\left(D^{3}+4 D\right) R a\right]^{2}$.

Equations (85) and (86) can then be rewritten:

$$
\begin{aligned}
& \partial_{T} W_{1}+H_{0}^{2} \frac{4 a^{\prime}}{\Pi} \nabla_{Z} W_{1}+H_{0}^{2} \frac{4 b^{\prime}}{\Pi} \nabla_{Z} W_{2}=\Delta W_{1}, \\
& \partial_{T} W_{2}+H_{0}^{2} \frac{4 c^{\prime}}{\Pi} \nabla_{Z} W_{1}+H_{0}^{2} \frac{4 d^{\prime}}{\Pi} \nabla_{Z} W_{2}=\Delta W_{2} .
\end{aligned}
$$

These formulas show that despite the zero helicity of the driving force, inside the system, an internal helicity is generated as a result of the joint impact of the Coriolis and buoyancy forces. This helicity plays an important role in the dynamics of the perturbations.

\subsection{Unstable and Oscillatory Modes in the Case of Negligible Viscosity $(k \ll 1)$}

In order to find instabilities, we choose the velocity $W_{1}, W_{2}$ in the form:

$$
\begin{aligned}
& W_{1}=\Upsilon_{1} \exp [i(k Z-\omega T)], \\
& W_{2}=\Upsilon_{2} \exp [i(k Z-\omega T)] .
\end{aligned}
$$

Injecting these solutions into (85), we obtain the simple system of equations:

$$
\begin{aligned}
& (a k-\omega) \Upsilon_{1}+b k \Upsilon_{2}=0, \\
& c k \Upsilon_{1}+(d k-\omega) \Upsilon_{2}=0 .
\end{aligned}
$$

Evidently we get a quadratic equation for $\omega$ :

$$
\omega^{2}-(a+d) k \omega+(a d-b c) k^{2}=0,
$$

which allows us to obtain the dispersion equations for the different modes.

\subsubsection{Dispersion Equation for the Unstable Mode}

This equation is obtained by searching for solutions of (96) for which the discriminant is negative, namely, $(a-d)^{2}+4 b c<0$. We show Figures 1 and 2 representing the area (in gray) of the plane $(D, R a)$ for which the discriminant is negative, this means that an instability can appear. Figure 1 shows the conditions for a negative temperature gradient and Figure 2, for a positive one. 


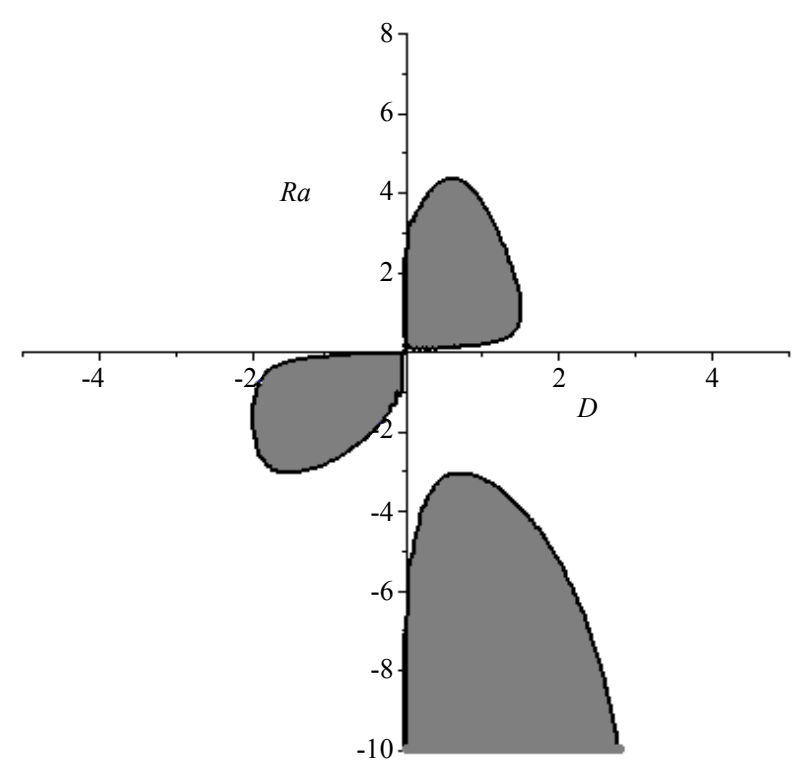

Figure 1. Instability condition with negative temperature gradient.

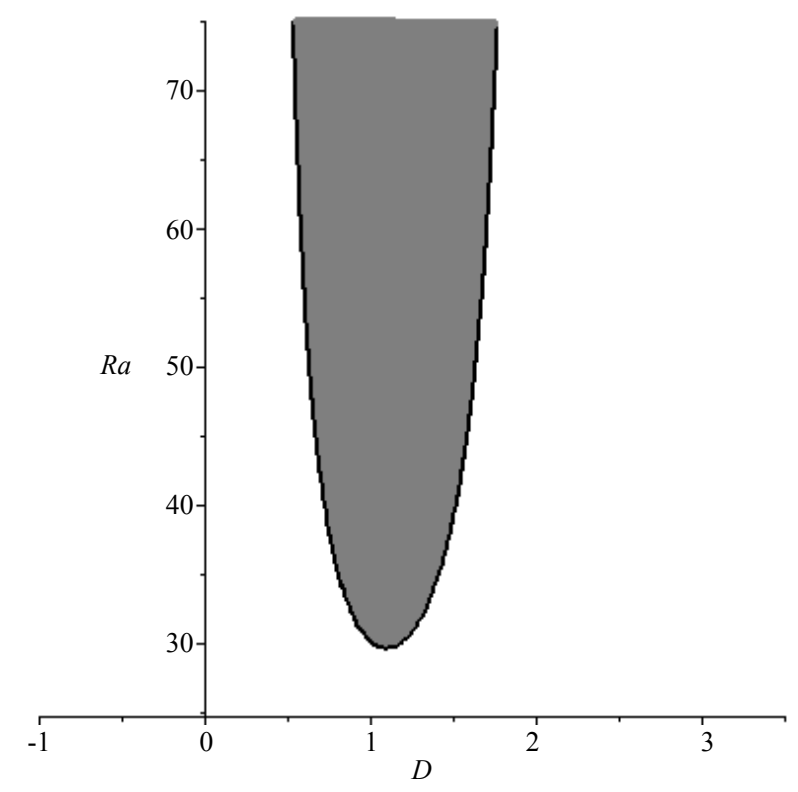

Figure 2. Instability condition with positive temperature gradient.

Finally, we get

$$
\begin{aligned}
& \omega=\omega_{0}(D, R a)+i \gamma(D, R a) \\
& =4 H_{0}^{2} \frac{a^{\prime}+d^{\prime}}{2 \Pi} k+i 4 H_{0}^{2} \frac{\sqrt{-\left[\left(a^{\prime}-d^{\prime}\right)^{2}+4 b^{\prime} c^{\prime}\right]}}{2 \Pi} k,
\end{aligned}
$$

where

$$
\gamma(D, R a)=4 H_{0}^{2} \frac{\sqrt{-\left[\left(a^{\prime}-d^{\prime}\right)^{2}+4 b^{\prime} c^{\prime}\right]}}{2 \Pi} k
$$

(97) is the growth rate of the instability. We note that it is proportionnal to the square of the helicity.

\subsubsection{Dispersion Equation for the Oscillatory Modes}

This Equation is obtained by searching for solutions of (96) for which the discriminant is positive, namely, $(a-d)^{2}+4 b c>0$.

We obtain in this case two oscillatory modes, $\omega_{1}$ and $\omega_{2}$, which are, respectively, a slow and a fast mode:

$$
\begin{aligned}
& \omega_{1}=4 H_{0}^{2} \frac{a^{\prime}+d^{\prime}-\sqrt{\left[\left(a^{\prime}-d^{\prime}\right)^{2}+4 b^{\prime} c^{\prime}\right]}}{2 \Pi} k, \\
& \omega_{2}=4 H_{0}^{2} \frac{a^{\prime}+d^{\prime}+\sqrt{\left[\left(a^{\prime}-d^{\prime}\right)^{2}+4 b^{\prime} c^{\prime}\right]}}{2 \Pi} k .
\end{aligned}
$$

It appears that both slow and fast oscillatory frequencies are proportional to the square of the helicity as well.

\subsection{Unstable and Oscillatory Modes with Viscosity}

In the same way as before, we get the system

$$
\begin{aligned}
& {\left[k^{2}+i(a k-\omega)\right] \Upsilon_{1}+i b k \Upsilon_{2}=0,} \\
& i c k \Upsilon_{1}+\left[k^{2}+i(d k-\omega)\right] \Upsilon_{2}=0 .
\end{aligned}
$$

We can then get a new quadratic Equation for $\omega$ :

$$
\begin{aligned}
& \omega^{2}+\left[2 i k^{2}-(a+d) k\right] \omega-k^{4}-i(a+d) k^{3} \\
& +(a d-b c) k^{2}=0 .
\end{aligned}
$$

\section{Dispersion Equation for the Unstable Mode}

The discriminant of this Equation is the same as in the nonviscous case, so the dispersion equation for the unstable mode has the same condition, namely $(a-d)^{2}$ $+4 b c<0$, which leads to:

$$
\begin{aligned}
\omega= & \omega_{0}(D, R a)+i \gamma(D, R a) \\
= & 4 H_{0}^{2} \frac{a^{\prime}+d^{\prime}}{2 \Pi} k \\
& +i\left(4 H_{0}^{2} \frac{\sqrt{-\left[\left(a^{\prime}-d^{\prime}\right)^{2}+4 b^{\prime} c^{\prime}\right]}}{2 \Pi} k-k^{2}\right),
\end{aligned}
$$

where

$$
\gamma(D, R a)=4 H_{0}^{2} \frac{\sqrt{-\left[\left(a^{\prime}-d^{\prime}\right)^{2}+4 b^{\prime} c^{\prime}\right]}}{2 \Pi} k-k^{2} .
$$

It is to be noted that the growth rate $\gamma(D, R a)$ is maximal for $k_{\max }=\frac{H_{0}^{2}}{\Pi} \sqrt{-\left[\left(a^{\prime}-d^{\prime}\right)^{2}+4 b^{\prime} c^{\prime}\right]}$, which can be considered as the characteristic scale of the gener- 
ated vortex structures. Below is Figure 3 showing the evolution of $\gamma$ with respect to the wave number $k$ for $D=R a=1$.

It can be noted that if the discrimant is positive, we get an oscillation with an exponentially decreasing amplitude.

With increasing amplitude, the instability becomes nonlinear and stabilizes. As a result, nonlinear vortex structures appear. The nonlinear stage of this instability and the results of numerical simulations will be presented in a future paper.

\section{Conclusions and Discussion of the Results}

In this paper, we showed that a large scale instability can appear in a rotating stratified fluid which is under the impact of a simple small scale external force (turbulence). The scale of this instability is much larger than the scale of the external force or turbulence. It is important to emphasize that, unlike previous papers about large scale instabilities, in the present paper, there are no special constraints imposed on the external force. It has a zero helicity and its parity needs not be violated; this means that this is a general force. Nevertheless, the small scale turbulence under the impact of the Coriolis force and the buoyancy force becomes helical. This helicity $H_{0}$, finally, is responsible for the generation of large scale instabilities because the growth rate $\gamma$ is proportional to $H_{0}^{2}$. The instability itself is oscillating while its frequency $\omega$ and $\gamma$ have, in principle, the same order. This means that the instability in the general case is aperiodic. The frequency of both the stable and unstable oscillations is also proportional to $H_{0}^{2}$. So we can say

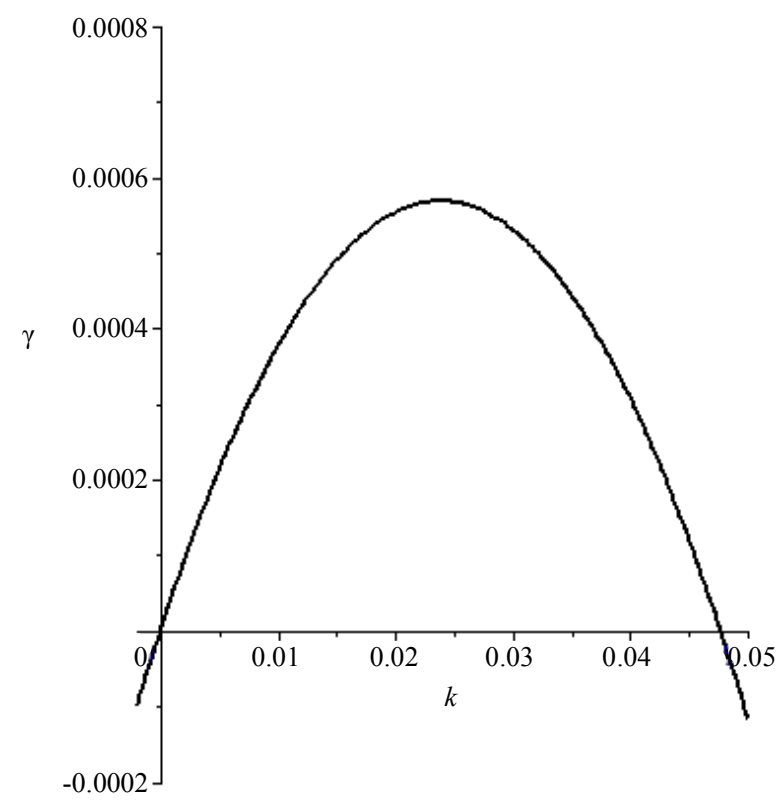

Figure 3. Evolution of the growth rate with respect to $k$. that the oscillation modes are inertial oscillations of the rotating fluid strongly modified by the helicity. There are two oscillating modes: one slow $\omega_{1}$ and one fast $\omega_{2}$. These oscillations decay when the viscosity is taken into account and in the case of instability, the maximal growth rate is reached at a characteristic scale of $k_{\max }$. Thereby this scale is typical for vortex structures like Beltrami's runaways. In this paper, the theory of a large scale instability was constructed using the method of multi-scale developments, which was proposed in the work of Frisch, She and Sulem [11]. The nonlinear secular equations for the large scale instability were obtained in the third order of development on a small Reynolds number. In this paper, we studied in detail the linear stage of the instability and the conditions of its appearance. It is interesting to note that instability is possible in the case of both stable and unstable stratifications. Moreover, that neither the Rayleigh number nor the Taylor number are assumed to be either big or small: this means that these numbers are out of scheme parameters. That is the reason why we should state that $R a<R a_{c r}$, where $R a_{c r}$ is the critical Rayleigh number for the generation of convective instability. The unstable stratification is typical for atmosphere dynamics while the stable one is typical for ocean dynamics. We believe that the instability which was found in this paper could be applied to the issue of the generation of large scale vortices in the atmosphere and the ocean, and to some astrophysical problems as well.

\section{REFERENCES}

[1] J. Sommeria, S. P. Meyers and H. L. Swinney, "Laboratory Simulation of Jupiter's Great Red Spot," Nature (London), Vol. 331, 1988, pp. 689-693. http://dx.doi.org/10.1038/331689a0

[2] G. Dritschel and B. Legras, "Modeling Oceanic and Atmospheric Vortices," Physics Today, Vol. 46, No. 3, 1993, p. 44. http://dx.doi.org/10.1063/1.881375

[3] J. C. McWilliams, "The Emergence of Isolated Coherent Vortices in Turbulent Flow," Journal of Fluid Mechanics, Vol. 146, 1984, pp. 21-43. http://dx.doi.org/10.1017/S0022112084001750

[4] J. Sommeria, "Experimental Study of the Two-Dimensional Inverse Energy Cascade in a Square Box," Journal of Fluid Mechanics, Vol. 170, 1986, pp. 139-168. http://dx.doi.org/10.1017/S0022112086000836

[5] R. H. Kraichnan, "Inertial Ranges in Two-Dimensional Turbulence," Physics of Fluids, Vol. 10, 1967, p. 1417. http://dx.doi.org/10.1063/1.1762301

[6] M. Chertkov, C. Connaughton, I. Kolokolov and V. Lebedev, "Dynamics of Energy Condensation in Two-Dimensional Turbulence," Physical Review Letters, Vol. 99, 2007, Article ID: 084501.

http://dx.doi.org/10.1103/PhysRevLett.99.084501

[7] D. Byrne, H. Xia and M. Shats, "Robust Inverse Energy 
Cascade and Turbulence Structure in Three-Dimensional Layers of Fluid," Physics of Fluids, Vol. 23, 2011, Article ID: 095109. http://dx.doi.org/10.1063/1.3638620

[8] Y. Couder and C. Basdevant, "Experimental and Numerical Study of Vortex Couples in Two-Dimensional Flows," Journal of Fluid Mechanics, Vol. 173, 1986, pp. 225-251. http://dx.doi.org/10.1017/S0022112086001155

[9] J. Paret and P. Tabeling, "Intermittency in the Two-Dimensional Inverse Cascade of Energy: Experimental Observations," Physics of Fluids, Vol. 10, No. 12, 1998, p. 3126. http://dx.doi.org/10.1063/1.869840

[10] D. Molenaar, H. J. H. Clercx and G. J. F. van Heijst, "Angular Momentum of Forced 2D Turbulence in a Square No-Slip Domain," Physica D, Vol. 196, No. 3-4, 2007, pp. 329-340. http://dx.doi.org/10.1016/j.physd.2004.06.001

[11] U. Frisch, Z. S. She and P. L. Sulem, "Large-Scale Flow Driven by the Anisotropic Kinetic Alpha Effect," Physica $D$, Vol. 28, No. 3, 1987, pp. 382-392. http://dx.doi.org/10.1016/0167-2789(87)90026-1

[12] P. L. Sulem, Z. S. She, H. Scholl and U. Frisch, "Generation of Large-Scale Structures in Three-Dimensional Flow Lacking Parity-Invariance," Journal of Fluid Mechanics, Vol. 205, 1989, p. 341. http://dx.doi.org/10.1017/S0022112089002065

[13] G. Rudiger, "On the $\alpha$-Effect for Slow and Fast Rotation," Astronomische Nachrichten, Vol. 299, No. 4, 1978, pp. 217-222. http://dx.doi.org/10.1002/asna.19782990408

[14] F. Krause and K.-H. Rädler, "Mean-Field Magnetohydrodynamics and Dynamo Theory," Akademie-Verlag, Berlin, 1980.

[15] H. K. Moffatt and A. Tsinober, "Helicity in Laminar and Turbulent Flow," Annual Review of Fluid Mechanics, Vol. 24, 1992, pp. 281-312. http://dx.doi.org/10.1146/annurev.fl.24.010192.001433

[16] S. S. Moiseev, R. Z. Sagdeev, A. V. Tur, G. A. Khomenko and V. V. Yanovsky, "A Theory of Large-Scale Structure Origination in Hydrodynamic Turbulence," Soviet Physics-JETP, Vol. 58, 1983, p. 1149.

[17] S. S. Moiseev, P. B. Rutkevich, A. V. Tur and V. V. Yanovsky, "Vortex Dynamos in a Helical Turbulent Convection," Soviet Physics-JETP, Vol. 67, 1988, p. 294.

[18] E. A. Lupyan, A. A. Mazurov, P. B. Rutkevich and A. V. Tur, "Generation of Large-Scale Vortices through the Action of Spiral Turbulence of a Convective Nature," Soviet Physics-JETP, Vol. 75, 1992, p. 833.
[19] G. A. Khomenko, S. S. Moiseev and A. V. Tur, "The Hydrodynamic Alpha-Effect in a Compressible Fluid," Journal of Fluid Mechanics, Vol. 225, 1991, pp. 355-369. http://dx.doi.org/10.1017/S0022112091002082

[20] R. Marino, P. D. Mininni, D. Rosenberg and A. Pouquet, "Emergence of Helicity in Rotating Stratified Turbulence," Physical Review E, Vol. 87, 2013, Article ID: 033016. http://dx.doi.org/10.1103/PhysRevE.87.033016

[21] Y. A. Berezin and V. P. Zhukov, "An Influence of Rotation on Convective Stability of Large Scale Distorbances in Turbulent Fluid," Izv. AN SSSR, Mech. Zhidk. Gaza, Vol. 4, 1989, p. 3

[22] P. B. Rutkevich, "Equation for Vortex Instability Caused by Convective Turbulence and the Coriolis Force," JETF, Vol. 77, 1993, p. 933.

[23] A. V. Tur and V. V. Yanovsky, "Non Linear Vortex Structures in Stratified Fluid Driven by Small-Scale Helical Force," Open Journal of Fluid Dynamics, Vol. 3, No. 2, 2013, pp. 64-74. http://dx.doi.org/10.4236/ojfd.2013.32009

[24] L. M. Smith and F. Waleffe, "Transfer of Energy to TwoDimensional Large Scales in Forced, Rotating ThreeDimensional Turbulence," Physics of Fluids, Vol. 11, No. 6, 1999, p. 1608. http://dx.doi.org/10.1063/1.870022

[25] L. M. Smith and F. Waleffe, "Generation of Slow Large Scales in Forced Rotating Stratified Turbulence," Journal of Fluid Mechanics, Vol. 451, 2002, pp. 145-168. http://dx.doi.org/10.1017/S0022112001006309

[26] B. Galanti and P. L. Sulem, "Inverse Cascades in ThreeDimensional Anisotropic Flows Lacking Parity Invariance," Physics of Fluids, Vol. A3, 1991, p. 1778.

[27] U. Frisch, "Turbulence: The Legacy of A. N. Kolmogorov," Cambridge University Press, Cambridge, 1995.

[28] F. Krause and G. Rudiger, "On the Reynolds Stress in Mean Field Hydrodynamics. 1. Incompressible Homogeneous Isotropic Turbulence," Astronomische Nachrichten, Vol. 295, No. 2, 1974, pp. 93-99. http://dx.doi.org/10.1002/asna.19742950205

[29] H. K. Moffat, "Magnetic Field Generation in Electrically Conducting Fluids," Cambridge University Press, Cambridge, 1978.

[30] G. V. Levina, S. S. Moiseev and P. B. Rutkevich, "Hydrodynamic Alpha-Effect in a Convective System," Advances in Fluid Mechanics, Vol. 25, 2000, p. 111. 


\section{Appendix}

\section{Calculation of the Reynolds Stress Tensor}

In order to calculate the Reynolds stress, we begin with the general expression

$$
\overline{v_{0}^{k} v_{0}^{i}}=T_{(1)}^{k i}+T_{(2)}^{k i}
$$

with

$$
T_{(1)}^{k i}=\overline{v_{01}^{k} v_{01}^{i *}}+\overline{v_{01}^{k *} v_{01}^{i}}
$$

and

$$
T_{(2)}^{k i}=\overline{v_{03}^{k} v_{03}^{i *}}+\overline{v_{03}^{k *} v_{03}^{i}} .
$$

Hence,

$$
T_{(1)}^{k i}=M_{k j(1)}^{-1} \frac{A^{j}}{D_{1}^{*}} \mathrm{e}^{i \varphi_{1}} \times M_{i t(1)}^{*-1} \frac{A^{* t}}{D_{1}} \mathrm{e}^{-i \varphi_{1}}+M_{k j(1)}^{*-1} \frac{A^{* j}}{D_{1}} \mathrm{e}^{-i \varphi_{1}} \times M_{i t(1)}^{-1} \frac{A^{t}}{D_{1}^{*}} \mathrm{e}^{i \varphi_{1}},
$$

and $T_{(2)}^{k i}$ has a similar expression.

Taking into account that only the components $A_{1}, A_{1}^{*}, B_{3}$ and $B_{3}^{*}$ of the external force are nonzero, and after some factorizations, we can write the two contribution of the Reynolds stress tensor in the following form:

$$
\begin{aligned}
T_{(1)}^{k i} & =\frac{\left(1+\frac{R a \hat{P}_{33}}{D_{1}^{* 2}}\right)}{\left|\Psi_{(1)}\right|^{2}\left|D_{1}\right|^{2}} A_{i} \xi_{k j(1)}^{*} A_{j}^{*}+\frac{\left(1+\frac{R a \hat{P}_{33}}{D_{1}^{2}}\right)}{\left|\Psi_{(1)}\right|^{2}\left|D_{1}\right|^{2}} A_{i}^{*} \xi_{k j(1)} A_{j}-\frac{D}{\left|\Psi_{(1)}\right|^{2}\left|D_{1}\right|^{2} D_{1}^{*}} \hat{P}_{i 2} A_{1} \xi_{k j(1)}^{*} A_{j}^{*} \\
& -\frac{D}{\left|\Psi_{(1)}\right|^{2}\left|D_{1}\right|^{2} D_{1}} \hat{P}_{i 2} A_{1}^{*} \xi_{k j(1)} A_{j}+\frac{1}{\left|\Psi_{(1)}\right|^{2}\left|D_{1}\right|^{2}}\left(\xi_{k j(1)} A_{j} \xi_{i t(1)}^{*} A_{t}^{*}+\xi_{k j(1)}^{*} A_{j}^{*} \xi_{i t(1)} A_{t}\right) .
\end{aligned}
$$

The same calculation for the contribution $T_{(2)}^{k i}$ gives us

$$
\begin{aligned}
T_{(2)}^{k i} & =-\frac{\left(1+\frac{R a \hat{P}_{33}}{D_{2}^{* 2}}\right) R a B_{3}^{*}}{\left|\Psi_{(2)}\right|^{2}\left|D_{2}\right|^{2} D_{2}^{2}} B_{k} \hat{P}_{i 3}-\frac{\left(1+\frac{R a \hat{P}_{33}}{D_{2}^{2}}\right) R a B_{3}}{\left|\Psi_{(2)}\right|^{2}\left|D_{2}\right|^{2} D_{2}^{* 2}} B_{k}^{*} \hat{P}_{i 3}+\frac{\left(1+\frac{R a \hat{P}_{33}}{D_{2}^{* 2}}\right)}{\left|\Psi_{(2)}\right|^{2}\left|D_{2}\right|^{2}} B_{k} \xi_{i t(2)}^{*} B_{t}^{*} \\
& +\frac{\left(1+\frac{R a \hat{P}_{33}}{D_{2}^{2}}\right)}{\left|\Psi_{(2)}\right|^{2}\left|D_{2}\right|^{2}} B_{k}^{*} \xi_{i t(2)} B_{t}+\frac{2 R a^{2} B_{3} B_{3}^{*}}{\left|\Psi_{(2)}\right|^{2}\left|D_{2}\right|^{6}}\left(\hat{P}_{i 3} \hat{P}_{k 3}\right)-\frac{R a B_{3}}{\left|\Psi_{(2)}\right|^{2}\left|D_{2}\right|^{2} D_{2}^{* 2}}\left(\hat{P}_{k 3} \xi_{i t(2)}^{*} B_{t}^{*}+\hat{P}_{i 3} \xi_{k j(2)}^{*} B_{j}^{*}\right) \\
& -\frac{R a B_{3}^{*}}{\left|\Psi_{(2)}\right|^{2}\left|D_{2}\right|^{2} D_{2}^{2}}\left(\hat{P}_{k 3} \xi_{i t(2)} B_{t}+\hat{P}_{i 3} \xi_{k j(2)} B_{j}\right)+\frac{1}{\left|\Psi_{(2)}\right|^{2}\left|D_{2}\right|^{2}}\left(\xi_{k j(2)} B_{j} \xi_{i t(2)}^{*} B_{t}^{*}+\xi_{k j(2)}^{*} B_{j}^{*} \xi_{i t(2)} B_{t}\right) .
\end{aligned}
$$

\section{Calculation of the Helicity}

The driving force has no helicity, but the joint action of the external force, Coriolis force, and the buoyancy give the internal helicity.

The general helicity of the velocity field $v_{0}$ is expressed by

$$
\begin{aligned}
H= & \overline{\boldsymbol{v}_{0} \cdot \nabla \times \boldsymbol{v}_{0}}=\overline{\boldsymbol{v}_{01} \cdot \nabla \times \boldsymbol{v}_{01}^{*}}+\overline{\boldsymbol{v}_{01}^{*} \cdot \nabla \times \boldsymbol{v}_{01}} \\
& +\overline{\boldsymbol{v}_{03} \cdot \nabla \times \boldsymbol{v}_{03}^{*}}+\overline{\boldsymbol{v}_{03}^{*} \cdot \nabla \times \boldsymbol{v}_{03}}=H_{(1)}+H_{(2)},
\end{aligned}
$$

where we choose $H_{(1)}$ and $H_{(2)}$ such that

$$
H_{(1)}=\overline{\boldsymbol{v}_{01} \cdot \nabla \times \boldsymbol{v}_{01}^{*}}+\overline{\boldsymbol{v}_{01}^{*} \cdot \nabla \times \boldsymbol{v}_{01}}
$$

and

$$
H_{(2)}=\overline{\boldsymbol{v}_{03} \cdot \nabla \times \boldsymbol{v}_{03}^{*}}+\overline{\boldsymbol{v}_{03}^{*} \cdot \nabla \times \boldsymbol{v}_{03}},
$$

and in indicial notation:

$$
\boldsymbol{v}_{0} \cdot \nabla \times \boldsymbol{v}_{0}=v_{0}^{k} \varepsilon_{k u i} \partial_{u} v_{0}^{i} .
$$

We must calculate $H$ with

$$
\begin{aligned}
H_{(1)} & =M_{k j(1)}^{-1} \frac{A^{j}}{D_{1}^{*}} \mathrm{e}^{i \varphi_{1}} \times \varepsilon_{k u i} \partial_{u}\left(M_{i t(1)}^{*-1} \frac{A^{* t}}{D_{1}} \mathrm{e}^{-i \varphi_{1}}\right) \\
& +M_{k j(1)}^{*-1} \frac{A^{* j}}{D_{1}} \mathrm{e}^{-i \varphi_{1}} \times \varepsilon_{k u i} \partial_{u}\left(M_{i t(1)}^{-1} \frac{A^{t}}{D_{1}^{*}} \mathrm{e}^{i \varphi_{1}}\right)
\end{aligned}
$$

$H_{(2)}$ is calculated in the same way, by replacing $v_{01}$ with $v_{03}$.

We finally obtain 


$$
\begin{aligned}
H= & \frac{D\left(1-W_{1}\right)\left[1+\left(1-W_{1}\right)^{2}\right]\left[2 R a-4\left[1+\left(1-W_{1}\right)^{2}\right]\right]}{L_{1}} \\
& -\frac{2 R a\left[\left[1-3\left(1-W_{1}\right)^{2}\right]-R a^{2}\right]}{L_{1}} \\
& +\frac{D R a\left(1-W_{2}\right)\left[2-6\left(1-W_{2}\right)^{2}+D^{2}\right]}{L_{2}} . \\
L_{1}= & {\left[D^{4}+R a^{2}+2 D^{2} R a+4\left[1+\left(1-W_{1}\right)^{2}\right]^{2}\right.} \\
& \left.+\left(2 D^{2}+2 R a\right)\left[2-2\left(1-W_{1}\right)^{2}\right]\right]\left[1+\left(1-W_{1}\right)^{2}\right]^{2} . \\
L_{2} & =\left[D^{4}+R a^{2}+2 D^{2} R a+4\left[1+\left(1-W_{2}\right)^{2}\right]^{2}\right. \\
& \left.+\left(2 D^{2}+2 R a\right)\left[2-2\left(1-W_{2}\right)^{2}\right]\right]\left[1+\left(1-W_{2}\right)^{2}\right]^{2} .
\end{aligned}
$$

After linearization:

$$
\begin{aligned}
H= & \frac{16 D^{5}+128 D^{3}-256 D-D R a^{4}-2 D^{3} R a^{3}}{\Lambda} W_{1} \\
& -\frac{\left(D^{5}+8 D^{3}+96 D\right) R a^{2}-\left(8 D^{5}+32 D^{3}-256 D\right) R a}{\Lambda} W_{1} \\
& +\frac{\left(D^{3}+8 D\right) R a^{3}+\left(2 D^{5}+8 D^{3}-32 D\right) R a^{2}}{\Lambda} W_{2} \\
& +\frac{\left(D^{7}+80 D^{3}\right) R a}{\Lambda} W_{2}+\frac{-16 D-D R a^{2}+\left(D^{3}+4 D\right) R a}{2 \sqrt{\Lambda}} .
\end{aligned}
$$

where we recall that $\Lambda=4\left(D^{4}+R a^{2}+2 D^{2} R a+16\right)^{2}$.

One can note that for small perturbations $\left(W_{1}, W_{2}\right)$, the helicity approaches the constant:

$$
H_{0}=\frac{-16 D-D R a^{2}+\left(D^{3}+4 D\right) R a}{2 \sqrt{\Lambda}},
$$

which can be considered as the internal helicity of the field $v_{0}$ when there are no perturbations. 